\title{
NOTE \\ Efficacité d'un paillage de papier journal sur la croissance initiale du merisier (Prunus avium L.)
}

\author{
H. FROCHOT * et G. LEVY** \\ avec la collaboration technique de L. WEHRLEN *, Y LEFEVRE **. G. LORSIN *** \\ *INRA, Station de Sylviculture et de Production \\ CNRF, Champenoux, 54280 Seichamps \\ *INRA, Station de Recherches sur le Sol, \\ la Microbiologie et la Nutrition des Arbres forestiers: \\ CNRF, Champenoux, 54280 Seichamps \\ *:* Boncourt-sur-Meuse, 55200 Commercy
}

\section{Résumé}

Un essai a été installé, juste après la plantation de merisiers, sur un sol brun calcaire bien préparé, en Lorraine. Six modalités sont étudiées : un témoin entretenu, un film plastique noir (référence) et quatre traitements mettant en ouvre un paillage de papier journal.

Deux ans après la mise en place, tous les traitements sont significativement supérieurs au témoin pour la hauteur et le diamètre des merisiers. Le meilleur traitement est, avec le film plastique noir, le journal ouvert paraffiné recouvert de terre. II permet de multiplier par trois l'accroissement en hauteur du merisier par rapport au témoin. Le journal disposé ì la surface du sol est assez bien conservé : il recouvre de l'ordre de 60 p. 100 de la surface initiale, mais avec une assez grande variabilité. Le journal enterré est encore bien moins altéré. du sol.

L'efficacité du papier journal semble être liée à une économie des réserves hydriques

Mots clés : Paillage, papier journal, croissance initiale, merisier.

\section{Introduction}

Le papier journal, installé sur le sol autour d'un jeune plant, permet-il d'améliorer la croissance initiale durant les premières années?

L'idée d'utiliser le papier comme paillage n'est pas nouvelle. Récemment, une firme suédoise a fabriqué un carton destiné à servir de paillage en zone aride : Hortopaper growing system (B.I.M.A., 1982).

Toutefois, le papier journal n'a pas, à notre connaissance, fait l'objet d'essais en tant que paillage. Son utilisation présenterait l'avantage : 
- de ne nécessiter aucune fabrication nouvelle;

- de mettre en auvre un matériel gratuit, et biodégradable, contrairement au plastique ;

- de trouver une solution élégante pour éliminer les vieux journaux.

On en attend une protection contre les mauvaises herbes et un effet favorable sur l'économie en eau du sol, comme dans les différents types de paillage. Il est intéressant de savoir comment il se situe par rapport au plastique noir, qui s'est déjà avéré généralement très efficace dans différents essais avec le merisier (FROCHOT \& LÉVY, 1980). C'est d'ailleurs pourquoi nous avons choisi le merisier dans ce premier essai destiné à tester la plus ou moins grande efficacité du papier journal en tant que paillage à la plantation.

\section{Matériel et méthode}

L'essai est réalisé à Boncourt-sur-Meuse, près de Commercy $(45 \mathrm{~km}$ à l'ouest de Nancy). Il est installé sur une pente moyenne, exposée au sud, en bordure de plateau calcaire, sur un sol brun calcaire caillouteux $(30$ p. 100 de cailloux en moyenne) de $60 \mathrm{~cm}$ de profondeur. C'est une ancienne friche remise en culture l'année précédant l'essai.

Les facteurs de variation choisis sont :

- la surface couverte : 1 ou 2 journaux ouverts, c'est-à-dire $60 \times 43 \mathrm{~cm}$ ou $60 \times 86 \mathrm{~cm}$;

- la préparation des journaux : paraffinés sur une face ou non. La paraffine pourrait permettre d'imperméabiliser les journaux et, éventuellement, d'en augmenter la durée de «fonctionnement $»$;

- leur mise en place : simplement tenus par des pierres, en surface de sol, ou recouverts de quelques centimètres de terre.

L'épaisseur d'application a été arbitrairement fixée à 10 feuilles, agrafées ensemble pour éviter la prise au vent. Le journal est fendu par le milieu pour permettre le passage de la tige. Lorsqu'il y a deux journaux, ils sont disposés de part et d'autre du plant, en se chevauchant légèrement.

Les traitements de référence sont, d'une part le paillage plastique noir, d'autre part un témoin désherbé. La surface du plastique équivaut à 2 journaux ouverts. qui sont :

On choisit un dispositif mono-arbre à 10 répétitions (blocs) et 6 modalités

- un journal en surface,

- deux journaux côte à côte en surface,

- deux journaux paraffinés côte à côte en surface,

- un journal paraffiné, recouvert de terre,

- un film de plastique noir en surface,

- un témoin désherbé entretenu. 
L'essai est installé sur sol propre (occupé, après défrichement, par une culture préparatoire de céréales pendant un an), et entretenu régulièrement par des engins agricoles; ainsi les conditions sont plus proches de l'agriculture que de la forêt. Des plants de merisier (Prunus avium L.) de 1 an (1-0) sont utilisés. La plantation a lieu à $2 \mathrm{~m}$ sur $3 \mathrm{~m}$, en mars 1983 , et les traitements sont appliqués immédiatement.

\section{Résultats}

Les mesures sur les plants ont été effectuées après 2 saisons de végétation.

Nous avons par ailleurs estimé visuellement 2 ans après la plantation le pourcentage de la surface initiale encore recouverte par du papier journal (ou du film plastique).

L'ensemble des résultats apparaît au tableau 1.

En ce qui concerne les plants, les classements des divers traitements pour l'accroissement en hauteur et en diamètre sont très comparables; les principaux résultats sont les suivants :

- le témoin est nettement inférieur à tous les autres traitements ;

\section{TABleau 1}

Résultats 2 ans après la plantation.

Les traitements qui n'ont pas au moins une lettre commune (a à d) sont statistiquement différents au seuil de $5 \mathrm{p} .100$.

Results 2 years after the plantation.

Treatments which do not have at least one letter (a to d) in common are statistically different.

\begin{tabular}{|c|c|c|c|c|c|c|}
\hline & Témoin & Journal & \begin{tabular}{l}
\multicolumn{1}{c}{ Deux } \\
journaux \\
paraffinés
\end{tabular} & $\begin{array}{c}\text { Deux } \\
\text { journaux } \\
\text { côte à côte }\end{array}$ & $\begin{array}{l}\text { Film } \\
\text { plastique } \\
\text { noir }\end{array}$ & $\begin{array}{l}\text { Journal } \\
\text { paraffiné } \\
\text { recouvert }\end{array}$ \\
\hline $\begin{array}{c}\text { Accroissement en hau- } \\
\text { teur }(\mathrm{cm}) \ldots \ldots \ldots \ldots\end{array}$ & $\begin{array}{c}34,9 \\
\text { a }\end{array}$ & $\begin{array}{c}71,9 \\
b\end{array}$ & $\begin{array}{c}81,0 \\
b c\end{array}$ & $\begin{array}{r}83,3 \\
\text { bcd }\end{array}$ & $\begin{array}{c}103,2 \\
\mathrm{~cd}\end{array}$ & $\begin{array}{c}106,5 \\
d\end{array}$ \\
\hline Diamètre $(\mathrm{mm})$ & $\begin{array}{c}11,4 \\
\mathrm{a}\end{array}$ & $\begin{array}{c}15,0 \\
b\end{array}$ & $\begin{array}{l}16,6 \\
\text { bc }\end{array}$ & $\begin{array}{l}16,3 \\
b c\end{array}$ & $\begin{array}{c}17,2 \\
b c\end{array}$ & $\begin{array}{c}18,1 \\
c\end{array}$ \\
\hline $\begin{array}{c}\text { Surface encore recouverte } \\
\text { par le papier journal } \\
\text { ou le plastique (p. 100) }\end{array}$ & & $\begin{array}{c}72 \\
\mathbf{a}\end{array}$ & $\begin{array}{c}57 \\
a\end{array}$ & $\begin{array}{c}56 \\
a\end{array}$ & $\begin{array}{c}100 \\
b\end{array}$ & $\begin{array}{c}90 \text { à } 100(*) \\
b\end{array}$ \\
\hline
\end{tabular}

(*) La surface restante des journaux n'a été estimée ici que sur une partie des répétitions, du fait des difficultés de décapage. - The remaining surface of the newspapers has been estimate here only on a part of the replications. 
- les 2 meilleurs traitements sont : le film plastique noir et le journal paraffiné recouvert de terre; ce dernier présente un accroissement en hauteur plus de 3 fois supérieur à celui du témoin ;

- le traitement à la paraffine n'améliore en rien l'efficacité des journaux disposés en surface. Le doublement de la surface recouverte par le papier journal semble augmenter la croissance du merisier, bien que les différences ne soient pas significatives dans cet essai.

Quant aux pourcentages de recouvrement en papier journal après 2 ans, la différence essentielle est apportée par la couverture de terre : elle permet aux journaux de rester en très bon état (et même encore parfaitement lisibles !), hormis quelques détériorations minimes sur le pourtour. Les journaux supportent assez bien les conditions climatiques locales. En ce qui concerne les journaux installés en surface, la pluie leur fait épouser la forme du terrain, le soleil les rend craquants, et ils présentent un aspect très variable selon les cas : parfois encore presque entiers, parfois réduits à quelques îlots de papier. Nous n'avons pas testé le journal recouvert de terre et non paraffiné; mais pour les journaux non enterrés, le paraffinage ne semble pas augmenter la «durée de vie ".

\section{Conclusion}

Un certain effet favorable du paillage à l'aide de papier journal était attendu : mais l'ampleur de cet effet est surprenant, en particulier le fait que les résultats, au bout de 2 ans, sont équivalents à ceux du paillage plastique noir, qui est considéré comme un traitement très efficace.

C'est très vraisemblablement l'économie des réserves hydriques du sol qui est en cause, au moins en très grande partie. Nous comptons vérifier prochainement cette hypothèse. A noter que le carton cité dans l'introduction (Hortopaper) réduirait de 30 p. 100 les pertes en eau. En plus d'une diminution de l'évaporation, le journal empêche l'herbe de se développer, du moins tant qu'il n'est pas trop détérioré, et si les espèces pérennes ont été préalablement supprimées.

Il reste, sur le plan pratique, à dégager une technique à la fois simple et efficace. C'est ainsi que le journal recouvert semble devoir être retenu prioritairement, en raison des effets constatés au bout de deux ans sur la croissance du merisier, mais aussi parce que l'altération du matériau est bien moins rapide que dans le cas d'une installation en surface. Le traitement à la paraffine accrôit la durée et le coût de la préparation des journaux, et son efficacité semble nulle, du moins dans le cas de journaux non recouverts. Nous vérifierons s'il en est de même sous une couverture de terre. Enfin, une surface correspondant à un journal simple (ouvert) semble devoir être retenue, en raison de la rapidité d'application, même si l'efficacité est un peu inférieure à celle d'une surface deux fois plus grande.

Il conviendra de tester, par ailleurs, différentes épaisseurs de papier journal (durée de vie, efficacité), ainsi que l'intérêt de ce type de paillage dans d'autres sols, sur d'autres espèces, et dans des conditions plus «forestières»; il faudra aussi évaluer le danger éventuel créé par l'encre d'imprimerie. Mais, on peut dès à présent penser que le papier journal, déchet disponible gratuitement, pourra être utilisé en appli- 
cation autour des plants pour activer la croissance initiale de certaines plantations. A titre indicatif, le rectangle unitaire de plastique noir utilisé dans cette expérience est revenu à environ $2 \mathrm{~F}$, et la durée des manipulations nécessaires à sa mise en œuvre est du même ordre de grandeur que celle des journaux enterrés, si l'on ne tient pas compte du paraffinage.

\section{Summary}

Effectiveness of a mulching with newspapers on juvenile growth of wild cherry-tree (Prunus avium L.)

An experiment has been set up in Lorraine on a brown calcareous soil well prepared, just after plantation. Six treatments have been compared : an (open) newspaper (10 sheet thick, $60 \mathrm{~cm} \times 43 \mathrm{~cm}$ ), two newspapers, a waxed newspaper, à waxed covered with soil newspaper, a black plastic film (treatment very effective), and a control kept without weeds.

Two years later, all treatments are significantly better than control for the height and diameter of the trees. The best treatment is, as well as the black plastic film, the newspaper covered with soil : it results in a height increment multiplied by 3 compared with the control.

The newspaper laid upon the soil surface is fairly well preserved : it covers about 60 p. 100 of the initial surface, but the variation is wide depending on the replications. The covered newspaper is still much less damaged.

The effectiveness of mulching with newspapers seems to be the result of a saving of the soil water supplies.

Key words: Mulching, newspaper, initial growth, wild cherry-tree.

\section{Références bibliographiques}

B.I.M.A., 1982. N" 986, p. 6.

Frochot H., Levy G., 1980. Facteurs limitants de la croissance initiale d'une plantation de merisier (Prunus avium L.) sur rendzine brunifiée. Ann. Sci. For., 37 (3), 239-248. 